\title{
VORLÄUFIGE ERGEBNISSE ÜBER DAS MESSEN DER SCHLACHTEIGENSCHAFTEN BEIM SCHWEIN
}

\author{
Unto Uusisalmi \\ Universität Helsinki, Institut für Haustierzüchtung
}

Eingegangen am 9.1.1969

Erster Teil der Untersuchung über die Schlachteigenschaften des Schweines begann ich im Jahre 1965 und den ihm unmittelbar folgenden Zweiten Teil 1966. Zweck der Untersuchung war es, 1) die verschiedenen Methoden zur Bestimmung des Schlachtwertes der lebendigen Schweine wie auch der Schlachtkörper miteinander zu vergleichen und 2) den Anteil der erblichen Variation an der Gesamtvariation jeder Eigenschaft zu schätzen.

Im ersten Teil der Untersuchung wurden bei 236 Stammversuchsschweinen für jedes etwa 200 Züge der Schlachtbeschaffenheit gemessen oder mit Punkten geschättz (Abb. 1). Die Messungen wurden mit Ultraschallgerät, Messband und Messlatte ausgeführt.

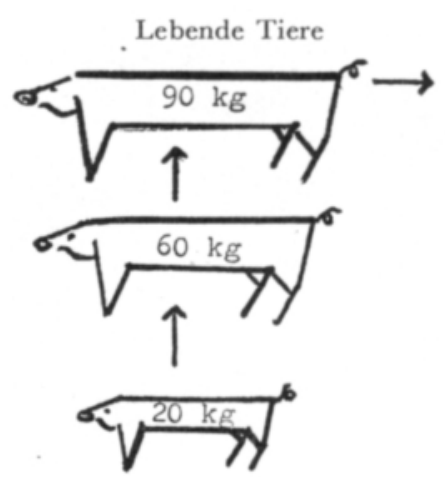

Schlachtkörper

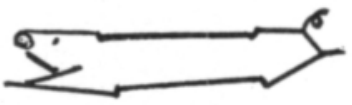

Abb. 1. Jedes Schwein wurde in den Lebendgewichtsklassen von 20, 60 und $90 \mathrm{~kg}$ bewertet und gemessen. 24 Stunden nach den letzten Messungen wurden die Tiere geschlachtet und 20 Stunden danach die Schlachtkörper bewertet.

\section{Bearbeitung der Ergebnisse}

Die Resultate wurden 1967 mit der Datenverarbeitungsmaschine Elliot 503 folgendermassen behandelt:

Die Korrelationsmatrix wurde für 179 gemessene und geschätzte Züge berechnt.

Nach der Methode der kleinsten Quadrate (Least squares analysis; Harvey 1966) 
wurde jede Variable zweimal bearbeitet. So analysierte man die auf die Gesamtvariation jeder Eigenschaft einwirkenden Faktoren, zu denen Lebendgewicht im Augenblick der Messung, Messungsquantitäten, Rasse, Geschlecht und Vatereber gehörten. Der Verfasser hat zuvor die Methode (Uusisalm 1967) in seiner pro gradu-Arbeit und Varo (1968) in seiner Untersuchung über Feldbewertung von Ebern für künstliche Besamung angewandt.

Als folgende wurde die wählende Regressionsanalyse für die Berechnung einiger als

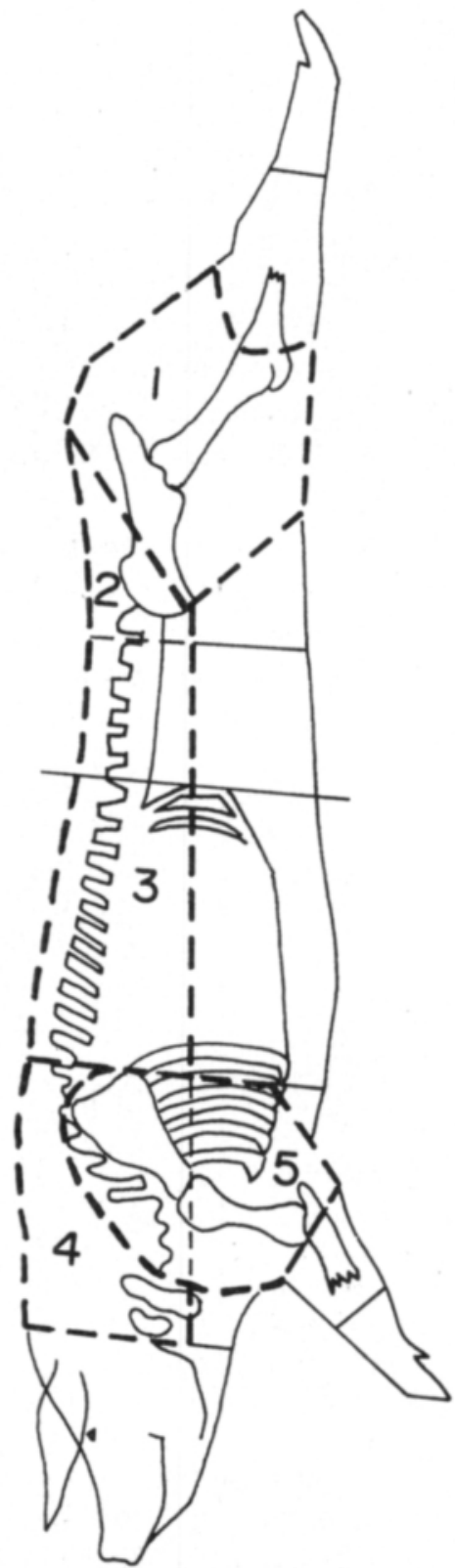

Abb. 2.'Von dem durch eine gestrichelte Linie begrenzten wertvollsten Abschnitt der Schlachtkörperhälfte sind durch Dissezieren Fet $\mathrm{t}$ u d $\mathrm{H}$ a $\mathrm{u} \mathrm{t}$ als die eine Komponente sowie F l e i s c h und $\mathrm{K}$ no chen als die andere voneinander getrennt worden.

Disseziert werden:

1 Schinken, 2 hinterer Schmorbraten, 3 Kotelett mit Rückenspeck, $4 \mathrm{Kamm}$ und 5 Bug. Die genannten Teile machen $62 \%$ vom Gewicht der Schlachtkörperhälfte aus und bilden etwa $3 / 4$ von dem im Einzelhandel zu erhaltenden Verkaufspreis der Schlachtkörperhälfte. 
wichtig erachteter Erklärungsmodelle benutzt. Die Methode ist in Verfassers pro graduArbeit (Uusisalmi 1967) beschrieben. Jede Analyse umfasst jeweils 50 Variable.

Ferner erfolgte eine Faktorenanalyse, in die 100 auf Grund früherer Bearbeitungen gewählte Eigenschaften oder Unveränderliche einbezogen waren.

VARo $(1962,1965)$ hat in seinen Untersuchungen festgestellt, dass die Ergebnisse der Faktorenanalyse von Gebrauchswert sind, wenn man in einer grossen Menge von Eigenschaften für die Zuchtwahl nach geeigneten Grundfaktoren sucht.

\section{Das Zerlegen der Schlachtkörper}

Die erster Phase der Untersuchung ermittelte, dass die herkömmliche Schlachtkörper bewertung als Grundlage einer derartigen mit der Züchtung verbundenen Erforschung der Schlachtkörperqualität nicht mehr ausreicht. So wurden in Zweiten Teil der Untersuchung ein ziemlich weitgehendes Zerlegen und Dissezieren der Schlachtkörper vorgenommen, um die relativen Mengen ihrer Teile zu klären sowie die Mengen von Fett, Fleisch und Knochen zu messen. Es wurde angestrebt, die Erfahrungen von Deutschen (Weniger et al. 1963) und Dänen (Pedersen 1964, 1967) in Betracht zu ziehen.

Die Ergebnisse von Zweiten Teil der Untersuchung sind erst teilweise behandelt worden. Die Bearbeitung ist zur Hauptsache auf gleiche Weise wie bei der erste Teil vor sich gegangen.

Auf Abb. 2 ist das auf Grund der vorläufigen Behandlung von mir entworfene Zerlegungsschema dargestellt. Vom wertvolls te $\mathrm{n} \mathrm{A} \mathrm{b} \mathrm{c} \mathrm{hnit} \mathrm{ein} \mathrm{e} \mathrm{r} \mathrm{S} \mathrm{c} \mathrm{h} \mathrm{la} \mathrm{ch} \mathrm{t-}$ körperhälfte sind $\mathrm{Haut}+\mathrm{Fet} \mathrm{t} \mathrm{als} \mathrm{der} \mathrm{eine} \mathrm{Teil} \mathrm{sowei} \mathrm{Fle} \mathrm{is} \mathrm{ch} \mathrm{+} \mathrm{K} \mathrm{n} \mathrm{o-}$ $\mathrm{c}$ h e n als der andere geschnitten worden. Der wertvollste Teil der Schlachtkörperhälften besteht aus dem Schinken, drei Rückenteilen und dem Bug. Er macht über $60 \%$ vom

Abb. 3

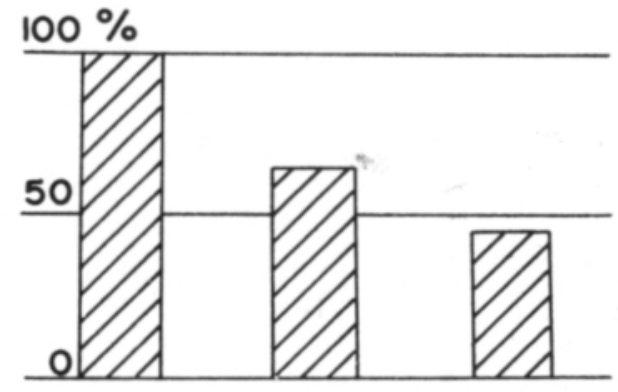

$\begin{array}{lll}\text { Hälfte des } & \text { Wertvollster } & \text { Fleisch }+ \\ \text { Schlachtkörpers } & \text { Teil der } & \text { Knochen des } \\ 100 & \text { Hälfte 62 } & \text { wertvollsten } \\ \text { Gewichts- } \% & \text { Gewichts-\% } & \text { Teiles 45 } \\ & & \text { Gewichts- } \%\end{array}$

Gewicht der Schlachtkörperhälfte aus (Abb. 3), und er bildet etwa 3/4 des im Einzelhandel zu erhaltenden Verkaufspreises der Schlachtkörperhälfte $(\mathrm{n}=97)$. Bei der Berechnung sind die von KanNari (1965) für die verschiedenen Teile des Schlachtkörpers darge- 
stellten Preiskoeffizienten und als Gewichte der verschiedenen Teile des Schlachtkörpers die für dieses Material berechneten durchschnittlichen Gewichte benutzt worden.

Experimentell werden nach dem dargestellten Schema schon die Tiere von fünf Versuchsstationen für Schweinezucht in Finnland zerlegt. Daneben wird noch die hergebrachte Schlachtkörperbewertung ausgeübt. So wird man wohl eine dem Bedarf der nächsten Zeit entsprechende Schlachtkörperbewertung zu entwickeln vermögen.

\section{Ergebnisse}

Die Ultraschallmessungen bei lebenden Schweinen. Nach den Untersuchungen RrTtLers (1968) verbessert die auf Grund des Alters vorgenommene Berichtigung der Rückenspeckkmasse neben der nach dem Gewicht erfolgten Korrektur die Messungsergebnisse nicht nennenswert. Da dies auch meiner Auffassung entspricht, beschränkte ich mich in der vorliegenden Untersuchung darauf, nur die Wirkung des Gewichts auf die Masse des Speckes zu analysieren.

Im ersten Teil meiner Untersuchung wurde an lebenden Schweinen der Speck bei den Lebendgewichtsklassen von $60 \mathrm{~kg}$ wie auch $90 \mathrm{~kg}$ gemessen und in Teil II meiner Untersuchung nur bei der Lebendgewichtsklasse von $90 \mathrm{~kg}$. Mit einer Berichtigung nach dem Lebendgewicht hätte man bei der Klasse von $90 \mathrm{~kg}$ nicht sehr viel weitere Information erhalten, weil der Anteil der linearen Regression nur 0-3\% der Variation der Speckmasse von Rücken und Brustseite ausmachte. Dagegen hätte bei den Klassen von $60 \mathrm{~kg}$ Lebendgewicht eine gewichtsmässige Berichtigung die Messungsergebnisse merklich zu verbessern vermocht, denn der Anteil der linearen Regression belief sich auf $10-23 \%$. Das von mir gewonnene Ergebnis lässt sich dadurch erklären, dass die Gewichtsunterschiede zwischen den Tieren bei der Klasse von $90 \mathrm{~kg}$ Lebendgewicht am geringsten waren. Ausserdem sei festgestellt, dass u.a. nach Отто (1967) die Speckzunahme bei $40-60 \mathrm{~kg}$ Lebendgewicht am stärksten und zugleich auch weniger linear ist. Dagegen wächst der Rückenspeck linear bei (60-) 70-110 kg Lebendgewicht (u.a. RITTLER 1966 und WENIGER et al. 1967). Somit dürfte es wenigstens unter Feldverhältnissen begründet sein, sich darauf zu beschränken, die Tiere bei $70-110 \mathrm{~kg}$ Lebendgewicht zu messen.

Tabelle 1. Das Korrelieren der Ultraschallmasse von Rücken mit Rücken- und Seitenspeck der Schlachtkörperbewertung (118 Borche und 118 Sauen).

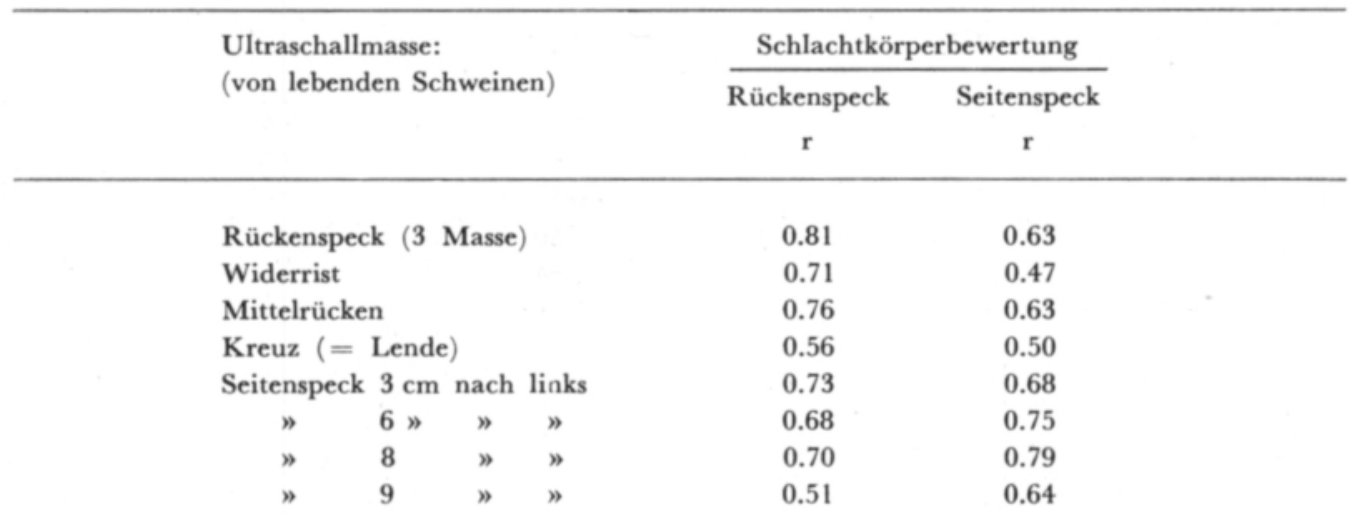


In Tabelle 1 ist das phänotypische Korrelieren der bei $90 \mathrm{~kg}$ Lebendgewicht erhaltenen Ultraschallmasse-von Rücken mit dem Rücken- und dem Seitenspeck zu sehen. Es kann festgestellt werden, dass die von mir ausgeführten Messungen in ihrer Genauigkeit dem Stand der ausländischen Untersuchungen entsprechen dürften. Die Korrelationskoeffizienten wechseln in Tabelle 1 zwischen 0.47 und 0.81. Der höchste Korrelationskoeffizient (0.81) hat sich zwischen dem Mittelwert dreier Ultraschallmessungen des Rückens und dem aus 5 Speckmessungen der Schlachtbewertung berechneten Rückenspeck ergeben. Doch lohnt es sich anzuführen, dass der Korrelationskoeffizient zwischen dem mit dem Ultraschallgerät erhaltenen Seitenspeck und dem durch die Schlachtkörperbewertung herausgestellten 0.79 betrug und dass die Ultraschallmasse über dem Rückenmuskel allgemein recht eng mit dem Seitenspeck der Schlachtkörperbewertung korrelierten.

Hier sei nur festgestellt, dass man mit den bei der Klasse von $60 \mathrm{~kg}$ Lebendgewicht aufgenommen Ultraschallmassen die Schlachtqualität nicht ebenso gut wie mit den bei der Klasse von $90 \mathrm{~kg}$ Lebendgewicht bestimmten Massen vorherzusagen vermag.

Die mit dem Ultraschallgerät erhaltenen Speckmasse korrelierten beinahe ebenso gut wie die durch Schlachtkörperbewertung bekommenen entsprechenden Masse (Tabelle 2) mit der Fett + Haut-Komponente des wertvollsten Teiles der Schlachtkörperhälfte. Die

Tabelle 2. Das Korrelieren der Speckmasse der Schlachtkörperbewertung und der mit Ultraschallgerät bei lebenden Schweinen erhaltenen Speckmasse mit der Komponente Fett + Haut und der Komponente Fleisch + Knochen des wwertvollsten Teiles der Schlachtkörperhälfte». Bei den Messungen 97 (69 Yorkshire +28 Landschweine) weibliche Tiere vertreten.

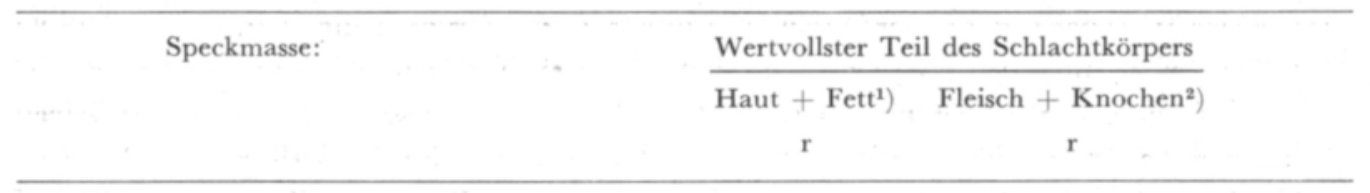

$\begin{array}{lll}\text { Aus der Schlachtkörperbewertung } & & \\ \text { Rückenspeck } & 0.72 & -0.46 \\ \text { Seitenspeck (Sol) } & 0.69 & -0.31 \\ \text { Widerrist } & 0.52 & -0.45 \\ \text { Mittelrücken } & 0.62 & -0.34 \\ \text { Kreuz (= Lende) } & 0.71 & -0.37 \\ & & \\ \text { Ultraschallmasse } & & -0.32 \\ \text { Seitenspeck (Sol) } & 0.60 & -0.31 \\ \text { Widerrist (Bug 0) } & 0.47 & -0.41 \\ \text { Bug cm links } & 0.56 & -0.33 \\ \text { "12 " " } & 0.61 & -0.36 \\ \text { Mittelrücken } & 0.50 & -0.38 \\ \text { Kreuz (Lende) } & 0.50 & -0.29 \\ \text { Lende } 6 \text { cm links } & 0.54 & -0.33 \\ \text { " } 12 \text { " " } & 0.48 & -0.35 \\ \text { " } 15 \text { " } & 0.59 & -0.18\end{array}$

1) Fett + Haut von Schinken, Bug und Rückenteilen sowie Nierenfett/2.

2) Fleisch + Knochen von Schinken, Bug und Rückenteilen. 
Korrelationskoeffizienten bleiben nur um 0.05-0.17 Einheiten geringer als bei der Messung lebender Schweine.

Die wählende Regressionsanalyse als Hilfsmittel beim Entwurfdes Messungsmodells fürden Rückenspeckund des Z e rlegungsmodells für den Schlach tkörper. Bei der traditionellen Schlachtkörperbewertung der sog. Stammversuchsschweine hat man den Rückenspeck als das beste Mass für den Fettgehalt des Schlachtkörpers angesehen. Deswegen wird im folgenden versucht, den Rückenspeck durch Punktbewertung der lebendigen Tiere, durch verschiedene äussere Masse und durch Ultraschallgeräte vorherzusagen. Insgesamt wurden 30 verschiedene Bewertungen und Masse als selektive Erklärer benutzt. - Mit der Datenverarbeitungsmaschine ist mit Hilfe der wählenden Regressionsanalyse aus den selektiven Erklärern in 8 Schritten das beste Modell für den Rückenspeck der Schlachtkörperbewertung aufgebaut worden (Tabelle 3). Bei jedem Schritt ist derjenige selektive Erklärer

Tabelle 3. Die Zunahme des mittels der wählenden Regressionanalyse erhaltenen multiplen Korrelationskoeffizienten (R) nach Schritten. Dominierende Funktion = Rückenspeck der Schlachtkörperbewertung. Selektive Erklärer sind die etwa einen Tag vor dem Schlachten an lebenden Schweinen $(\mathrm{n}=236)$ aufgenommenen Ultraschall- u.a. Masse sowie die Punktbewertung, insgesamt 30 Werte.

\begin{tabular}{llccc}
\hline $\begin{array}{l}\text { Schritt } \\
\text { Nr. }\end{array}$ & Um einen Schritt vermehrter & F-Zahl & \multicolumn{2}{c}{ Nach der Zunahme erhalten } \\
\cline { 3 - 4 } & Erklärer & & $\mathrm{R}$ & $\mathrm{R}^{2}$ \\
\hline & & & & 0.573 \\
1 & Speck vom Mittelrücken & $316.15^{* * *}$ & 0.757 & 0.686 \\
2 & Speck vom Widerrist & $84.06 * * *$ & 0.828 & 0.726 \\
3 & Seitenspeck 6 & $35.85 * * *$ & 0.852 & 0.736 \\
4 & Speck vor der Lende (Lendenmass 1) & $10.07 * *$ & 0.858 & 0.743 \\
5 & Kopflänge & $7.38^{* *}$ & 0.862 & 0.748 \\
6 & Muskeltiefe 6 & $5.65 *$ & 0.865 & 0.750 \\
7 & Seitenspeck 8 (Sol) & 3.81 & 0.866 & 0.753 \\
8 & Seitenspeck 9 & 3.00 & 0.868 &
\end{tabular}

angegeben, der den multiplen Korrelationskoeffizienten $(=\mathrm{R})$ zwischen der dominierenden Funktion und den bei den früheren Schritten einbezogenen selektiven Erklärern am meisten gesteigert hat.

Das berechnete Modell hat in acht Schritten 6 durch ein Ultraschallgerät erhaltene Speckmasse, 1 durch ein Ultraschallgerät gemessene Muskeldicke und 1 äusseres Mass, die Kopflänge, umfasst. Dies dürfte die wichtige Stellung des Ultraschallgeräts bei dem Bemühen, ein dünnspeckiges Schwein zu wählen, beweisen. Auch mag es begründet sein, zu bemerken, dass in das Modell 3 eigentliche auf dem Rücken festgestellte Masse und 3 über dem Rückenmuskel aufgenommene Speckmasse eingehen. Dadurch dürfte schon bewiesen werden, dass beim Bestimmen des Speckgehaltes beim lebendigen Schwein Messungen sowohl auf dem Rücken als auch über dem Rückenmuskel erforderlich sind. - Schon mit den drei ersten Schritten hat man etwa $73 \%\left(\mathrm{R}^{2}=0.726\right)$ der Gesamtvarianz des Rückenspeckes zu erklären vermocht, und die danach erhaltene zusätzliche Information ist verhältnismässig belanglos. 
Die Fläche des Querschnittes des langen Rückenmuskels (= M. long. dorsi) hat man beim lebendigen Tier dagegen merklich schwächer als die ebengenannte vorhersagen können. Doch sei hier angeführt, dass die Tiefe des Muskels mit dem Ultraschallgerät denn auch nur an 3 Stellen gemessen worden ist, nämlich in der Höhe des hinteren Randes der letzten Rippe in Abständen von 3, 6 und $9 \mathrm{~cm}$ vom Mittelrücken auf die linke Seite. Die wählende Regressionsanalyse vermag durch 9 Schritte nur $28 \%$ von der Gesamtvarianz der Muskelfläche zu erklären. In das Erklärungsmodell wurden 5 Ultraschallmasse einbezogen, von denen 4 ein Speckmass und 1 ein Muskelmass war. Hinzu kamen an äusseren Massen Breite bei den Hüften, Mass hinter den Bugen und Schlachtkörperumfang. An Punkten wurden die für den Schinken mit berücksichtigt. Das obenbeschrieben für die Vorhersage der Muskelfläche konstruierte Modell war imstande, immerhin $50 \%$ von der Gesamtvarianz des Rückenspeckes der Schlachtkörperbewertung zu erklären.

Hier kann festgestellt werden, dass für die Bestimmung der Muskelfläche einige mit Ultraschallgerät aufgenommene Tiefenmasse des Muskels nicht zu genügen scheinen. Offenbar wäre es auch unerlässlich, für die Dauer der Messung das Tier in einem Gestell festhalten.

Zum Schluss stelle ich eine von mir beobachtete Anwendungsmöglichkeit der wählenden Regressionsanalyse dar. Dabei ist versucht worden, aus den über die Dissektion des Schlachtkörpers gewonnenen Ergebnissen ein für Schlachthofverhältnisse geeignetes, mit geringer Arbeit verbundenes Zerlegungsmodell (z.B. das Modell von Abb. 1) aufzustellen, durch das man jedoch eine möglichst reichhaltige Kenntnis des Schlachtkörperwertes zu erlangen versucht (als Beispiel Tabelle 4). Soweit Haut, Fett, Fleisch und Knochen der

Tabelle 4. Die Zunahme des durch wählende Regressionsanalyse nach Schritten erhaltenen multiplen Korrelationskoeffizienten (R). Dominierende Funktion $=$ Fett + Haut des wertvollsten Schlachtkörperteils. Selektive Erklärer sind die Komponenten Fett + Haut sowie die Komponenten Fleisch + Knochen (zus. 10 St.) vom Schinken, Bug, Kotelett, Kamm und hinteren Schmorbraten. $\mathbf{n}=97$.

\begin{tabular}{|c|c|c|c|c|c|c|}
\hline \multirow{2}{*}{$\begin{array}{l}\text { Schritt } \\
\text { Nr. }\end{array}$} & \multirow{2}{*}{\multicolumn{3}{|c|}{$\begin{array}{l}\text { Um einen Schritt vermehrter } \\
\text { Erklärer }\end{array}$}} & \multirow[t]{2}{*}{ F-Zahl } & \multicolumn{2}{|c|}{ Nach der Zunahme erhalten } \\
\hline & & & & & $\mathbf{R}$ & $\mathbf{R}^{2}$ \\
\hline 1 & Fett + Haut & vom & Kotelett & $243.78 * * *$ & 0.847 & 0.717 \\
\hline 2 & $" \quad n$ & $"$ & Schinken & $74.87 * * *$ & 0.917 & 0.841 \\
\hline 3 & $"$ & $"$ & Kamm & $62.31 * * *$ & 0.951 & 0.904 \\
\hline 4 & $"$ & $"$ & Bug & $40.06 * * *$ & 0.965 & 0.931 \\
\hline 5 & $"$ & $"$ & hinteren & & & \\
\hline & Schmorbrate & & & $42.69 * * *$ & 0.976 & 0.953 \\
\hline
\end{tabular}

einzelnen Schlachtkörperteile auseinander geschnitten sind, kann für jede Gewebeart ihr eigenes Messungsmodell mit Hilfe der wählenden Regressionsanalyse aufgestellt werden.

In Tabelle 4 ist dargestellt, wie man durch die wählende Regressionsanalyse die Komponente Fe t t $+\mathrm{H}$ a u t des wertvollsten Teiles des Schlachtkörpers zu erklären vermocht hat. Auf gleiche Weise ist auch die Komponente Fle i s c h $+\mathrm{K}$ n o c h e n vom wertvollsten Teil des Schlachtkörpers erhellt worden. In beide Analysen waren die gleichen 
10 selektiven Erklärer einbezogen worden, nämlich die Teile $\mathrm{H}$ a u $\mathrm{t}+\mathrm{F}$ e $\mathrm{t} \mathrm{t}$ und die Teile $\mathrm{Fle}$ is ch $+\mathrm{Kn}$ o chen von Schinken, Rückenpartien und Bug.

Aus Tabelle 4 ist zu ersehen, dass in das Modell $\mathrm{Fe} \mathrm{t} \mathrm{t}+\mathrm{H}$ a u t des wertvollsten Schlachtkörperteils in den drei ersten Schritten die komponenten $\mathrm{F}$ e t t $+\mathrm{H} \mathrm{a} \mathrm{u} \mathrm{t}$ von Schinken, Kotelett-Abschnitt und Kamm aufgenommen worden sind. Des weiteren sei angeführt, dass in das Modell $\mathrm{Fle}$ i s $\mathrm{ch}+\mathrm{K} \mathrm{n}$ o c h e $\mathrm{n}$ in den drei ersten Schritten die Komponenten Fleis ch $+\mathrm{K}$ n o chen der gleichen Schlachtkörperteile eingegangen sind. Die Variation in der Menge von $\mathrm{Fet} \mathrm{t} \mathrm{und} \mathrm{Ha}$ a des wertvollsten Schlachtkörperteils wie auch die Gesamtvarianz der Menge von Fleis ch und $\mathrm{K}$ n o c h e n sind durch Zerschneiden der genannten drei Teile mit über $90 \%$ herausgestellt worden. Die übrigen, hier noch nicht dargestellten Analysen erweisen u.a., dass schon durch Zerlegen von Schinken und Kotelett über Speck und Fleischgehalt des wertvollsten Schlachtkörperteils ein besseres Bild als durch die gesamte Schlachtkörperbewertung erhalten worden ist.

\section{Zusammenfassung}

Als Zusammenfassung über die bisherigen Ergebnisse kann dargestellt werden:

Die subjektive Punktbewertung ist unsicher als Indikator der Schlachtkörperbeschaffenheit und vor allem des Fettgehalts sowie der Muskelstärke des Schlachtkörpers. Desgleichen sind unsichere Indikatoren der Schlachtkörperbeschaffenheit die an lebenden Tieren aufgenommenen äusseren Masse (z.B. Längen-, Breiten-, Höhen- und Umfansmass).

Die Ultraschallmessungen sind das wichtigste und auch für praktische Verhältnisse gut geeignete Verfahren, die Dicke des Speckes zu bestimmen. Als Beispiel sei angeführt, dass der mit einem Ultraschallgerät an 236 Schweinen gemessene Rückenspeck diese Komponente Schlachtkörpers $(\mathrm{r}=0.81)$ und ebenfalls der am lebenden Tier gemessene Seitenspeck ebendiesen Anteil am Schlachtkörper $(r=0.79)$ gut vorhersagte. Eine wirksame Auswertung des Ultraschallgeräts bei Bestimmung des Phänotyps bedeutet eine Intensivierung der Züchtungsarbeit zugunsten der Schlachtkörperbeschaffenheit (Jonsson 1967), eine beträchtliche Zunahme in der Anzahl der in den Kreis der Auslese gelangenden Tiere und zugleich eigentlich die einzige brauchbare Weise, die Säue mit in die Zuchtwahl einzubeziehen.

Bei diesem Stand kommt man dazu, den Fleischgehalt des Schlachtkörpers nur durch teilweise Zerlegung und Dissektion der Schlachtkörper befriedigend zu messen. Die Untersuchung erweist u.a., dass durch Zerschneiden des Schinkens sowie des Kotelettabschnittes in Fett + Haut als den einen Teil und in Fleisch + Knochen als den anderen Teil über die Komponente Fett + Haut wie auch die Komponente Fleisch + Knochen der wertvollsten Partie der Schlachtkörperhälfte ein besseres Bild gewonnen worden ist als durch die gesamte herkömmliche Schlachtkörperbewertung $(\mathrm{n}=97)$.

Aus Tabelle 2 lässt sich ersehen, dass zwischen den Korrelationskoeffizienten des Teiles Fett + Haut und des Teiles Fleisch + Knochen ein ziemlich schwacher Zusammenhang besteht. Die Folge davon dürfte sein, dass man bei sich verdünnendem Rückenspeck des Schweines dazu kommt, bei der Züchtung gerade dem Messen der Fleischeigenschaften des Tieres stets grössere Aufmerksamkeit zuwendet, weil der Verringerung der Fettmenge 
des Schlachtköpers eine in gleicher Grössenordnung erfolgende Zunahme der Fleischmenge gar nich mehr entspricht. Bei Erforschung des ausgelesenen Stammversuchsmaterials dürften wir uns schon in dieser Situation befinden.

Für den Teil des statistischen Verarbeitungssystems scheinen die Ergebnisse vielversprechend. Im Anschluss an einen zweckmässigen Versuchsplan vermittelt das automatisierte Datenverarbeitungssystem bei den mit Fleischfragen verbundenen Untersuchungen brauchbare Kenntnis:

Mit Hilfe der Faktorenanalyse werden die der Forschung als Gegenstand vorliegenden Variablen oder Eigenschaften zu Merkmalgruppen zusammengefasst.

Durch wählende Regressionsanalysen werden die Modelle für jede in der Gruppe dominierende Eigenschaft oder eine aus der Sicht der Untersuchung sonstwie interessante Eigenheit aufgebaut.

Die Gesamtvariation jeder gemessenen Eigenschaft oder jedes gemessenen Zuges wird nach der Methode der kleinsten Quadrate analysiert. So erhält man die Bewertung des relativen Anteils der Umwelt- und der erblichen Faktoren an der Gesamtvariation jeder Eigenschaft.

\section{LITERATUR}

Bö́ckenhoff, E., Fewson, D. \& Bischoff, T. 1967. Die wirtschaftliche Bedeutung verschiedener Leistungsmerkmale beim Schwein und ihre Auswirkung auf die Zuchtarbeit. 1. Mitteilung. Z-kunde 39: 270282.

HARVeY, W. R. 1966. Least-squares analysis of data with unequal subclass numbers. ARS 20-8 July 1960. Reprinted with corrections April 1966. Agric. Res. Service U. S. Dept. of Agric.

Jonsson, P. 1967. Modelberegning over selektionsmetoder i svineavlen på grundlag af forsegsresultaterne på de fire faste svineforsegstationer i Danmark. Fortrykk av foredrag NJF-kongressen 27.-30. juni 1967.

KANNARI, M. 1965. Miksi paisti on kallista. Osuusteurastamo Nr. 4: 7.

Отто, E. 1967. Rückenspeckzunahme bei Schweinen im Gewicht von 40 bis $115 \mathrm{~kg}$. Tierzucht $21: 602$. Pedersen, O. K. 1964. Bestemmelse af svinens slagtekvalitet. Forsøgslaboratoriets årbog: $257-267$.

—»- 1967. Bedømmelsescentraler for forsegssvin. Landøkonomisk Forsøgslaboratoriums efterårsmøde. Årbog 1967: 171-178.

Ritrler, A. 1966. Untersuchungen über Echolotmessungen als Hilfsmittel für die Schätzung des Zuchtwertes junger Eber. Z-kunde 38: $11-21$.

—— 1968. Echolotmessungen als Hilfsmittel für die Zuchtwertschätzung beim Schwein. 2. Mitteilung.

s. f. Tierz. u. z. - Biol. 84: 179-187.

Standal, N. 1962. A Scoring-System for Pig Selektion based on Performance Testing for Backfat Thickness and Rate of Gain. (Særtrykk av) Meldinger fra Norges Landbrukshøgskole Nr. 4, 1962.

Uusısalmı, U. 1966. Ultraääni avuksi jalostukseen. Koetoim. ja Käyt. 23: 9.

—- 1966. Sikojen ruokinnanohjaus ja tutkaaja. Ibid. 23: 42.

—— 1967. Sian teurasominaisuuksien periytymisestä ja mittaamisesta. Pro gradu-Arbeit (unveröffentlicht).

—— 1968. Mitä silavaluotaus on — ja mikä vaikuttaa sillä saatuihin tuloksiin. Sika Nr. 2: 6-8.

$\longrightarrow$ - 1968. Sian teurasominaisuuksien mittaamisesta ja periytymisestä. Ibid. Nr. 4: 10-12, 14, $17-19$.

Valikoiva regressioanalyysi (= Wählende Regressionsanalyse). Valtion tietokonekeskus, Suunnitteluosasto $\times 40$ 1578: 26. 2. 1966. EVL/KH 503 tiedoituslehti: 42 r-v.

VAro, M. 1962. Über die Begrenzung der Beurteilungseigenschaften bei der Eberaus[ese. Ergebnis der Faktorenanalyse. Ann. Agr. Fenn. 1: 267-283.

- - 1965. Einige Beobachtungen über Anwendungsmöglichkeiten von Ergebnissen der Faktorenanalyse. Ibid. 4: $91-95$. 
VAro, M. 1967. Preliminary results of the "field testing" of A. I. Boars. Acta Agr. Fenn. 109: 105-109. —- 1968. On the progeny-testing of boars in "field conditions». J. Sci. Agr. Soc. Finland 40: $109-113$.

—— \& Partanen, J. 1965. Bewertung der Heritabilität von einigen Eigenschaften bei Schweinen. Ann. Agr. Fenn. 4: 46-48.

Weniger, J. H., Kallwett, E., Steinhauf, D. \& Glodek, P. 1967. Einfluss des Wachstums auf Teilstückgewichte und Schlachtkörpermasse beim Schwein, Z-kunde 39: 188-199.

-”- , Steinauf, D. \& PAhl, G. H. M. 1963. Muskeltopographie der Schlachtkörper. BLV Verlagsgesellschaft München, Basel, Wien.

\title{
SELOSTUS
}

\section{ENNAKKOTULOKSIA SIAN TEURASOMINAISUUKSIEN MITTAAMISESTA}

\author{
Unto Uusisalmi
}

\section{Helsingin yliopiston kotieläinten jalssiustieteen laitos}

Tutkimus perustuu mittauksiin, joita on tehty Puistolan sikatalouskoeaseman kantakoesioista vuosina 1965 - 67. Tämän tutkimuksen tarkoitukset olivat seuraavat.

Pyrittiin vertailemaan eri menetelmiä määrittää teurasarvoa sekä elävistä sioista että teurasruhoista. Elävät siat arvosteltiin subjektiivisesti pistein sekä tehtiin erilaisia mittauksia mm. ultraäänilaitteella. Samojen sikojen ruhot arvosteltiin normaalisti kantakoearvostelussa sekä suoritettiin lisäksi eräitä mittauksia. Tutkimuksen jälkimmäisessä osassa ruhoja vielä paloiteltiin ja luotiin kuvan 2 osoittama paloittelusysteemi.

Tutkimuksessa pyrittiin selvittämään perinnöllisen muuntelun osuutta kunkin ominaisuuden kokonaismuuntelusta pienimmän neliösumman metelmällä (HARVEY 1966).

Tietokonetta pyrittiin käyttämään hyväksi mahdollisimman monipuolisesti mittaustulosten käsittelyssä.

Lyhyenä yhteenvetona tuloksista voidaan esittää $\mathrm{mm}$. seuraavaa. Subjektiivinen pistearvostelu on epävarma teuraslaadun osoittajana. Samoin ovat epävarmoja elävistä eläimistä otetut ulkoiset mitat. Ultraäänimittaukset on tärkein ja käytännön olosuhteisiinkin hyvin soveltuva keino määrittää ruhon rasvaisuutta. Esimerkkinä mainittakoon, että ultraäänilaitteella mitattu selkäsilava (236 sikaa) ennusti hyvin teurasruhon selkäsilavaa $(r=0.81)$ ja samoin elävästä mitattu kylkisilava teurasruhon kylkisilavaa $(r=$ 0.79). Sen sijaan tässä vaiheessa ruhon lihapitoisuutta päästään mittaamaan vain ruhojen osittaisella paloittelulla. Tutkimus osoittaa (leikattiin 97 ruhon puoliskoa), että leikkaamalla kinkun ja kyljyksen rasva + nahka toiseksi osaksi ja liha + luut toiseksi osaksi saatiin ruhon puoliskon rasva + nahka-komponentista samoin kuin liha + luut-komponentista parempi kuva kuin koko traditionaalisella teurasarvostelulla. Selitykseksi tuli yli $\mathbf{8 0} \%$ ruhon puoliskon rasva + nahka-komponentista samoin kuin liha + luut-komponentistakin.

Tilastollisen käsittelyjärjestelmän osalta tulokset näyttävät lupaavilta. Tarkoituksenmukaiseen koesuunnitteluun yhdistettynä saadaan seuraavalla ATK-järjestelmällä lihakysymyksiin liittyvissä tutkimuksissa käyttökelpoista tietoa.

Faktorianalyysin avulla ryhmitellään tutkimuksen kohteena olevat ominaisuudet piirreryhmiksi.

Valikoivilla regressioanalyyseillä rakennetaan mallit kussakin ryhmässä esiintyvälle dominoivalle piirteelle tai tutkimuksen kannalta muuten mielenkiintoiselle piirteelle.

Kunkin mitatun piirteen kokonaismuuntelua analysoidaan pienimmän neliösumman menetelmällä. Näin saadaan arvioiduksi ympäristötekijäin ja perinnöllisten tekijäin suhteellinen osuus kunkin ominaisuuden kokonaismuuntelusta. 